\section{ECONOMICS}

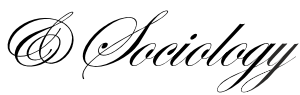

\title{
STRATEGIC MAP FOR HOSPITAL MANAGEMENT: PERSPECTIVES AND PRIORITIES
}

\author{
Andrzej Niemiec, \\ Poznan University of Economics, \\ Poznañ, Poland, \\ E-mail: \\ andrzej.niemie@@ue.poznan.pl
}

Received: December, 2015

1st Revision: March, 2016

Accepted: June, 2016

DOI: $10.14254 / 2071-$

789X.2016/9-3/6

JEL Classification: I11, M41, M21

\section{Introduction}

It seems certain that there will be more changes in the healthcare industry in the next 15 years then there has been in the past 15 years. Why? What is the source of change? First of all, healthcare institutions continue to face a turbulent, confusing, and often threatening environment. It means that one of the most well described source of the change is external (Luigi et al., 2014). Legislative and policy initiatives, changes in demographics, increasing prevalence of chronic diseases, changes in lifestyles, consumers' lack of trust in traditional health systems, demand for more sophisticated, convenient, transparent, affordable and personalized service as well as increasing costs on healthcare (as \% to GDP) have great impact on healthcare institutions Rudawska \& Pętlak, 2013). This trends are well described in literature, and continuously researched by organizations and institution like: PWC (2014, 2015), CDC (Bernstein et al., 2003), Premier Healthcare Alliance, individual scientists (Rudawska \& Urbańczyk, 2014) and practitioners.

But there are also other sources of change. Nowadays, healthcare institutions change in the way every organization does (Rudawska, 2013). One issue of this change is especially important for healthcare institutions because of their specificity. It is sustainable development. The concept of sustainable development represents a shift in understanding of humanity's place on the planet (Hopwood et al., 2005). Why it is so important for management of a healthcare institution? Mostly, because these organizations are predestinated to implement sustainable development in some parts world-wide. In some parts they are doing it already. The UN have identified 17 goals and 169 targets of sustainable development (UN, 2015). One of the main goals is to ensure healthy lives and promote well-being for all at all ages. Some of the targets are obvious: reduce the global maternal mortality ratio to less than 70 per 100,000 live births or preventable deaths of newborns and children under 5 years of age, with 
all countries aiming to reduce neonatal mortality to at least as low as 12 per 1,000 live births and under-5 mortality to at least as low as 25 per 1,000 live births. But some of them need additional effort. In particular, the epidemics of AIDS, tuberculosis, malaria and tropical diseases; combating hepatitis, water-borne diseases and other communicable diseases by the end of 2030 or reduce by one third premature mortality from non-communicable diseases through prevention and treatment and also, promoting mental health and well-being. Summarizing, healthcare institutions in future should become more open for the society, and more cooperative in fulfilling these goals.

For hospital management it becomes increasingly difficult to adapt to the pace of policy changes and reforms while balancing economical considerations, social responsibility to provide high-quality health care to the community served and, finally, ecological concerns (Glouberman, Mintzberg, 2001). In this context, hospital management focuses on understanding the interconnections on the macro level - between society and the ecosystem and changing its strategy to optimize the outcomes for these systems.

Sustainable development concept requires managing hospitals on three different fields using three different value systems. Economic aims requires economic calculation of revenues, costs, incomes and expanses. Social aims in hospital management requires considering social reflections i.e. impacts of various diseases on society (epidemiology). Ecologic aims requires also different calculation. Ecologic calculation is focused on hospital impact on the environment. It is connected with waste management, drugs usage, as well as bioethics issues (Fig. 1).

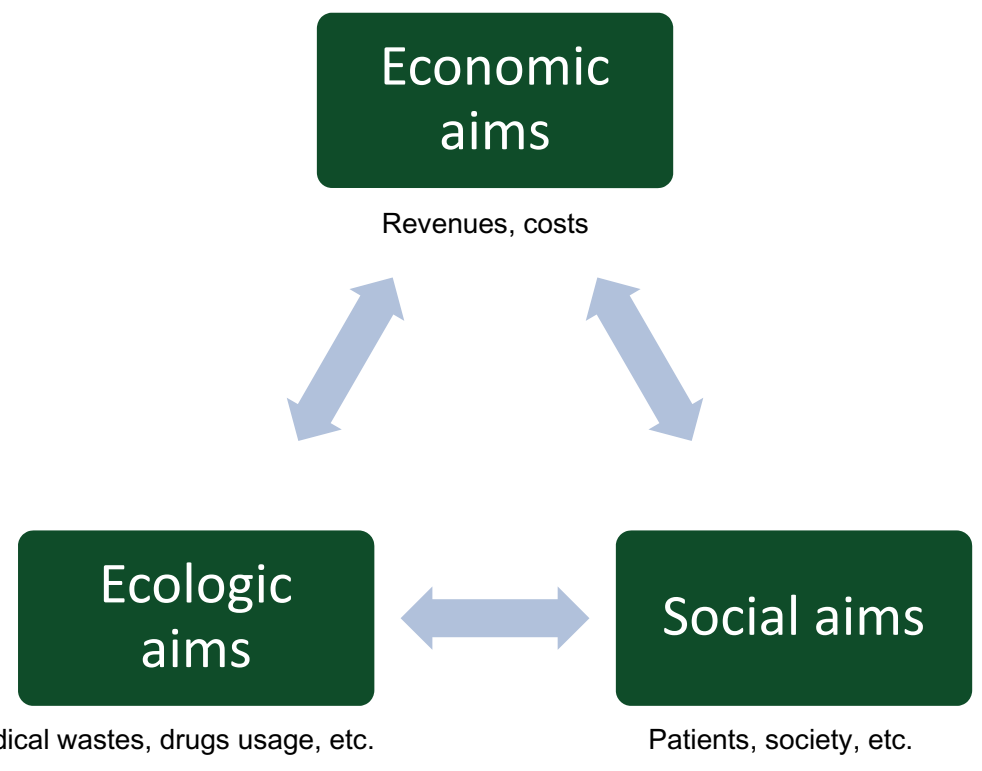

Figure 1. Sustainable development

Source: Wall, Schröder, 2009, p. 264.

Between economic, social and ecologic aspects here there is an obvious conflict. The main source of this conflict is the lack of resources, especially financial ones (Urbaniec, 2015). For such a large amount of objectives money would be always scarce. According to this conflict a hospital manager should prioritize the objectives. And a very useful tool in this case is strategic map. 
The aim of this article is to adopt strategic map to the hospital management context. Hospital as a business unit has needs very much different from other companies and/or corporations, different priorities, different fields of interests. The u use of strategic map here requires to be adopted first to hospital management specificity. In this article such an adaptation is carried out. Strategic y map is constructed for a functioning county hospital in Poland.

\section{Literature Review}

Nowadays, organizations needs the tools, which could be used to communicate strategy and responsibilities to the employees in a clear and transparent way. Strategy map could be such a tool (Świerk, 2009). Strategy maps show how an organization plans to convert its various assets into desired outcomes. The template shows how employees need certain knowledge, skills, and systems (learning and growth perspective) to innovate and build the right strategic capabilities and efficiencies (internal process perspective) so that they can deliver specific value to the market (customer perspective), which will lead to higher shareholder value (financial perspective). For the customer perspective, companies typically select one of three strategies: operational excellence, customer intimacy, or product leadership (Kaplan \& Norton, 2000). The objectives are grouped in perspectives and linked to each other. The sense of map strategy is graphic presentation of strategy. The strategic map connects all the objectives; in the same way, every action leads to reaction in daily clinical practice (Verzola et al., 2009).

A strategy map enables an organization to describe and illustrate, in clear and general language, its objectives, initiatives, and targets; the measures used to assess its performance (such as market share and customer surveys); and the linkages that are the foundation for strategic direction (Kaplan \& Norton, 2000). According to Kaplan \& Norton $(2000,2004)$ strategy map is an auxiliary tool for balanced scorecard. But can be used also as a separate strategic tool. The strategy map's value becomes evident when it is used as a tool that enables people to express and share strategic priorities; these two aspects can actually facilitate the difficult process of re-formulating a company's strategy and hence feeding the strategic learning loop (Biazzo \& Garengo, 2012).

If strategy map can be separate strategic tool, important question is if balanced scorecard is really needed in this case? Is balanced scorecard good tool for management count hospital? Is it possible to use strategy map with other strategic tools, like i.e. quality management model (Rudawska \& Kaufmann, 2015)? Balanced scorecard in hospitals have advantages and disadvantages (Tab. 1).

Table 1. Advantages and disadvantages of balanced scorecard in hospitals

\begin{tabular}{|c|c|c|}
\hline \multirow[b]{2}{*}{ Advantages } & \multicolumn{2}{|l|}{ Disadvantages } \\
\hline & Literature & $\begin{array}{l}\text { Practitioner opinion } \\
\text { ( } 7 \text { opinions) }\end{array}$ \\
\hline $\begin{array}{l}\text { In order to enable collaboration } \\
\text { between the various organizational } \\
\text { figures, it was necessary to form a } \\
\text { business strategy which was } \\
\text { accessible to the health workers } \\
\text { involved. } \\
\text { - It brings the business strategy to the } \\
\text { objectives attributed to each } \\
\text { operative unit, becoming a tool }\end{array}$ & $\begin{array}{l}\text { - It could be interpreted by health } \\
\text { workers as a system of measurement } \\
\text { proposed by the management and } \\
\text { thus as a potential work overload. } \\
\text { - One criticality that could be met by } \\
\text { the health workers when using such } \\
\text { a tool is linked to the difficulty in } \\
\text { finding specific indicators and/or } \\
\text { their related values as a direct }\end{array}$ & $\begin{array}{l}\text { - } \begin{array}{l}\text { Easy to } \\
\text { manipulate, }\end{array} \\
\text { - Focused more on } \\
\text { analytics then on } \\
\text { actions, } \\
\text { - Timeframe of } \\
\text { data depends on } \\
\text { information } \\
\text { collect process, }\end{array}$ \\
\hline
\end{tabular}


orientated to guide managerial action.

- Thanks to the BSC, the strategy may be communicated to all levels of the organization, and allocation of resources can reflect the strategic aims. consequence of the aforementioned lack of information.

- One factor which could potentially limit the reliability of results of this methodology may be represented by the objective difficulty in the choice or definition of reference standards, or benchmark.

Source: compiled by the authors and from Verzola et al., 2009.

Strategy map is free of balanced scorecard disadvantages, but have all its advantages. Moreover, it visualize objectives and linkage between them. Via the strategic map and the sequence of cause effect relationships, permitted explanation to all levels of the organization that, for example, improving the relationship with the users increases the patients' trust (Verzola et al., 2009).

Summarizing, strategy map:

- represents business model of organization;

- is a strategic tool, because represents objectives;

- is more user friendly then balanced scorecard with its tables or myriads of redyellow-green lights;

- can require additional management tools.

Originally strategy map have identical structure than balance scorecard. Groups objectives into four perspectives (Kaplan \& Norton, 2000):

- financial - typically starts with a financial strategy for increasing shareholder value (Nonprofit and government units often place their customers or constituents - not the financials - at the top of their strategy maps). Companies have two basic levers for their financial strategy: revenue growth and productivity.

- customer - the core of any business strategy is the customer value proposition, which describes the unique mix of product and service attributes, customer relations, and corporate image that a company offers. It defines how the organization will differentiate itself from competitors to attract, retain, and deepen relationships with targeted customers. The value proposition is crucial because it helps an organization connect its internal processes to improved outcomes with its customers.

- internal process - captures these critical organizational activities, which fall into four high-level processes: build the franchise by innovating with new products and services and by penetrating new markets and customer segments; increase customer value by deepening relationships with existing customers; achieve operational excellence by improving supply chain management, the cost, quality, and cycle time of internal processes, asset utilization, and capacity management; and become a good corporate citizen by establishing effective relationships with external stakeholders.

- learning and growth - defines the core competencies and skills, the technologies, and the corporate culture needed to support an organization's strategy. These objectives enable a company to align its human resources and information technology with its strategy. Specifically, the organization must determine how it will satisfy the requirements from critical internal processes, the differentiated value proposition, and customer relationships. Although executive teams readily 
acknowledge the importance of the learning and growth perspective, they generally have trouble defining the corresponding objectives.

In literature were many publications presenting implementation of balanced scorecard in health care institution (Tab. 2).

Table 2. Perspectives and number of metrics used in balanced scorecard

\begin{tabular}{|c|c|c|c|c|}
\hline Year & Who & Perspectives & $\begin{array}{c}\text { No. of } \\
\text { perspectives }\end{array}$ & $\begin{array}{l}\text { No. of } \\
\text { KPI }\end{array}$ \\
\hline 2002 & $\begin{array}{l}\text { Chang L., Lin S.W., } \\
\text { Northcott D.N. }\end{array}$ & $\begin{array}{l}\text { Health improvement, fair access, } \\
\text { effective service delivery, health } \\
\text { efficiency, patient experience, outcomes }\end{array}$ & 6 & 49 \\
\hline \multirow{2}{*}{2003} & Radnor Z., Lovell B. & $\begin{array}{l}\text { Client (government and users), learning } \\
\text { and growth, internal process, cost }\end{array}$ & 4 & 29 \\
\hline & Radnor Z., Lovell B. ${ }^{*}$ & $\begin{array}{l}\text { Client (government and users), learning } \\
\text { and growth, internal process, cost }\end{array}$ & 4 & 30 \\
\hline \multirow{2}{*}{2004} & $\begin{array}{l}\text { Huang S.H., } \\
\text { Chang W.Y., } \\
\text { Chen P.L., Yang M.C. }\end{array}$ & Business processes, financial, customer & 3 & 9 \\
\hline & $\begin{array}{l}\text { Asbroek A.H., } \\
\text { Arah O.A., Geelhoed J., } \\
\text { et al." }\end{array}$ & $\begin{array}{c}\text { Financial, consumer, internal business, } \\
\text { innovation }\end{array}$ & 4 & 20 \\
\hline \multirow{5}{*}{2005} & $\begin{array}{l}\text { Kunz H., Schaaf T., } \\
\text { Braun J., Tolxdorff T. }\end{array}$ & $\begin{array}{c}\text { Patient, social, financial, innovation, } \\
\text { process }\end{array}$ & 5 & \\
\hline & $\begin{array}{l}\text { Karra E.D., } \\
\text { Papadopoulos D.L. }\end{array}$ & $\begin{array}{l}\text { Internal process, learning and growth, } \\
\text { stakeholder (customer), management } \\
\text { (financial) }\end{array}$ & 4 & 16 \\
\hline & Kumar A., Ozdamar L. & $\begin{array}{l}\text { Customer, finance, process, learning } \\
\text { and growth }\end{array}$ & 4 & 8 \\
\hline & Smith H., Kim L. ${ }^{*}$ & $\begin{array}{l}\text { Quality, service, employee worklife, } \\
\text { financial, business growth }\end{array}$ & 5 & 23 \\
\hline & Urrutia I., Eriksen S.D. ${ }^{*}$ & $\begin{array}{l}\text { Patients, internal process, financiers } \\
\text { and/or political body, formation and } \\
\text { growth, environment, mission }\end{array}$ & 6 & 30 \\
\hline \multirow{7}{*}{2006} & $\begin{array}{l}\text { Walker KB., } \\
\text { Dunn L.M. }\end{array}$ & $\begin{array}{l}\text { Quality, access, customer satisfaction, } \\
\text { performance, efficiency }\end{array}$ & 5 & 18 \\
\hline & $\begin{array}{l}\text { Walker KB., } \\
\text { Dunn L.M. }\end{array}$ & $\begin{array}{l}\text { Share growth, quality and process } \\
\text { improvement, organizational health }\end{array}$ & 3 & 17 \\
\hline & $\begin{array}{l}\text { Van de Wetering R., } \\
\text { Batenburg R., } \\
\text { Versendaal J., } \\
\text { Lederman R., Firth L. }\end{array}$ & $\begin{array}{c}\text { Clinical business process, patient, } \\
\text { quality and transparency, information } \\
\text { systems }\end{array}$ & 4 & 12 \\
\hline & $\begin{array}{l}\text { Peters D.H., Noor A.H., } \\
\text { Singh L.P., et al. }\end{array}$ & $\begin{array}{l}\text { Patient and community, staff, capacity } \\
\text { for service provision, service provision, } \\
\text { financial system, overall vision }\end{array}$ & 6 & 29 \\
\hline & $\begin{array}{l}\text { Chen X.Y., } \\
\text { Yamauchi K., Kato K., } \\
\text { Nishimura A., Ito K. }\end{array}$ & $\begin{array}{l}\text { Financial, internal business processes, } \\
\text { customer, learning and growth }\end{array}$ & 4 & 19 \\
\hline & Coop C.F. ${ }^{*}$ & $\begin{array}{l}\text { Financial, clinical quality, productivity, } \\
\text { learning and organizational health }\end{array}$ & 4 & 21 \\
\hline & $\begin{array}{l}\text { Gonzalez M.E., } \\
\text { Quesada G., Urrutia I., } \\
\text { Gavidia J.V.* }\end{array}$ & $\begin{array}{l}\text { Health systems, patients, internal } \\
\text { process, support }\end{array}$ & 4 & \\
\hline
\end{tabular}




\begin{tabular}{|c|c|c|c|c|}
\hline \multirow{3}{*}{2007} & Schmidt S. ${ }^{*}$ & $\begin{array}{l}\text { Clinical risk, finance, service } \\
\text { modernization, workforce }\end{array}$ & 4 & 23 \\
\hline & $\begin{array}{l}\text { Radford A., Pink G., } \\
\text { Ricketts T. }\end{array}$ & $\begin{array}{l}\text { Access to care, financial performance, } \\
\text { human resources, utilization and } \\
\text { productivity }\end{array}$ & 4 & 19 \\
\hline & $\begin{array}{l}\text { De Toni A.F., } \\
\text { Fornasier A., } \\
\text { Montagner M., } \\
\text { Nonino F. }\end{array}$ & $\begin{array}{l}\text { Financial, final consumer/ customer, } \\
\text { facilities, learning and growth }\end{array}$ & 3 & 25 \\
\hline \multirow[b]{2}{*}{2008} & Josey C., Kim IW. $^{*}$ & People, service quality, finance, growth & 4 & 27 \\
\hline & Hong, et al. & $\begin{array}{l}\text { Financial, internal business processes, } \\
\text { customer, learning and growth }\end{array}$ & 4 & 29 \\
\hline 2009 & $\begin{array}{l}\text { Groene, O., Brandt, E., } \\
\text { Schmidt, W., Moeller J. }\end{array}$ & $\begin{array}{c}\text { Internal processes, learning \& growth, } \\
\text { clients, financial }\end{array}$ & 4 & $\begin{array}{c}\min . \\
20\end{array}$ \\
\hline \multirow{3}{*}{2010} & $\begin{array}{l}\text { Rabbani F., } \\
\text { Jafri S.M.W., Abbas F., } \\
\text { et al. }\end{array}$ & $\begin{array}{l}\text { Financial, internal business process, } \\
\text { human resource, patient satisfaction }\end{array}$ & 4 & 20 \\
\hline & Lovaglio P.G. & $\begin{array}{c}\text { Human capital, clinical process, patient } \\
\text { satisfaction, economy }\end{array}$ & 4 & 25 \\
\hline & $\begin{array}{l}\text { Steinke C., Webster L., } \\
\text { Fontaine M. }\end{array}$ & Service, functional, physical, financial & 4 & \\
\hline \multirow{4}{*}{2011} & Kollberg B., Elg M. ${ }^{*}$ & $\begin{array}{l}\text { Financial, process, employees, } \\
\text { innovation and development, customer }\end{array}$ & 5 & 25 \\
\hline & $\begin{array}{l}\text { Grigoroudis E., } \\
\text { Orfanoudaki E., } \\
\text { Zopounidis C. } \\
\end{array}$ & $\begin{array}{l}\text { Financial, internal business processes, } \\
\text { customer, learning and growth }\end{array}$ & 4 & 24 \\
\hline & $\begin{array}{l}\text { Bouland D.L., Fink E., } \\
\text { Fontanesi J. }\end{array}$ & $\begin{array}{c}\text { Quality and safety, customer } \\
\text { satisfaction, physician/staff satisfaction, } \\
\text { finance }\end{array}$ & 4 & $\min .4$ \\
\hline & $\begin{array}{l}\text { Rabbani F., } \\
\text { Lalji S.N.H., Abbas F., } \\
\text { et al. }\end{array}$ & $\begin{array}{l}\text { Financial, internal business, human } \\
\text { resource, patient satisfaction }\end{array}$ & 4 & 18 \\
\hline \multirow{6}{*}{2012} & $\begin{array}{l}\text { Chen H.F., Hou Y.H., } \\
\text { Chang R.E. }\end{array}$ & $\begin{array}{l}\text { Clinical utilization and outcomes, } \\
\text { financial performance and condition, } \\
\text { system integration and human } \\
\text { resources, patient satisfaction }\end{array}$ & 4 & 21 \\
\hline & Wu I.L., Kuo Y.Z. ${ }^{*}$ & $\begin{array}{l}\text { Financial, internal business processes, } \\
\text { customer, learning and growth }\end{array}$ & 4 & 38 \\
\hline & $\begin{array}{l}\text { Chen H.F., Hou Y.H., } \\
\text { Chang R.E. }\end{array}$ & $\begin{array}{l}\text { Finance, administration, admission } \\
\text { performance, quality of care }\end{array}$ & 4 & 9 \\
\hline & $\begin{array}{l}\text { Lovaglio P.G., } \\
\text { Vittadini G. }^{*}\end{array}$ & $\begin{array}{l}\text { Human capital, patient satisfaction, } \\
\text { clinical process, economy }\end{array}$ & 4 & 21 \\
\hline & $\begin{array}{l}\text { Cattinelli I., Bolzoni E., } \\
\text { Barbieri C., et al. }\end{array}$ & $\begin{array}{c}\text { Patient, employee, shareholder, } \\
\text { community }\end{array}$ & 4 & 30 \\
\hline & $\begin{array}{l}\text { Grigoroudis E., } \\
\text { Orfanoudaki E., } \\
\text { Zopounidis C. }\end{array}$ & $\begin{array}{l}\text { Financial, internal business processes, } \\
\text { customer, learning and growth }\end{array}$ & 4 & 24 \\
\hline \multirow[b]{2}{*}{2013} & $\begin{array}{l}\text { Lin Q.L., Liu L., } \\
\text { Liu H.C., Wang D.J. }\end{array}$ & $\begin{array}{l}\text { Financial, internal business processes, } \\
\text { customer, learning and growth }\end{array}$ & 4 & 32 \\
\hline & $\begin{array}{l}\text { Khan M.M.R., } \\
\text { Hotchkiss D., } \\
\text { Dmytraczenko T., } \\
\text { Zunaid A.K. }\end{array}$ & $\begin{array}{l}\text { Capacity forservice provision, human } \\
\text { resource-related capacity, delivery of } \\
\text { healthcare services, patient or client }\end{array}$ & 4 & 37 \\
\hline
\end{tabular}




\begin{tabular}{|c|c|c|c|c|}
\hline & $\begin{array}{l}\text { Abo-Hamad W., } \\
\text { Arisha A. }\end{array}$ & Efficiency, patient throughput & 2 & 16 \\
\hline \multirow{3}{*}{2014} & $\begin{array}{l}\text { Ermakova S.E., } \\
\text { Izmalkov N.S., } \\
\text { Evdokimova E.G. }\end{array}$ & $\begin{array}{l}\text { Internal processes, learning \& growth, } \\
\text { clients, financial }\end{array}$ & 4 & 19 \\
\hline & Szabo S., Sidor J. & $\begin{array}{c}\text { Employees, processes, customer, } \\
\text { finance }\end{array}$ & 4 & \\
\hline & $\begin{array}{l}\text { Smith C., } \\
\text { Christiansen T., } \\
\text { Dick D., et al. }\end{array}$ & $\begin{array}{l}\text { Efficient, safe, appropriate, accessible, } \\
\text { acceptable, effective }\end{array}$ & 6 & 8 \\
\hline \multirow{5}{*}{2015} & $\begin{array}{l}\text { Gutiérrez López C., } \\
\text { Culebras J.L.M.J. }\end{array}$ & $\begin{array}{c}\text { Internal processes, learning \& growth, } \\
\text { clients, financial }\end{array}$ & 4 & 20 \\
\hline & Baraldi S., Cifalinò A. & $\begin{array}{l}\text { Organizational, trainee, internal } \\
\text { process, learning and growth }\end{array}$ & 4 & 42 \\
\hline & $\begin{array}{l}\text { Rabbani F., } \\
\text { Pradhan N.A., Zaidi S., } \\
\text { Azam S.I., Yousuf F. }\end{array}$ & $\begin{array}{l}\text { Health facility functionality, service } \\
\text { provision, staff capacity, staff and } \\
\text { patient satisfaction }\end{array}$ & 5 & 20 \\
\hline & $\begin{array}{l}\text { Noh W., } \\
\text { Seomun GA. }\end{array}$ & $\begin{array}{c}\text { Internal processes, learning \& growth, } \\
\text { clients, financial }\end{array}$ & 4 & 31 \\
\hline & $\begin{array}{l}\text { Marquez P.B., Barroso } \\
\text { C.C.A., Silva A.M. } \\
\text { et al. }\end{array}$ & $\begin{array}{l}\text { Internal processes, learning \& growth, } \\
\text { clients, financial }\end{array}$ & 4 & 16 \\
\hline
\end{tabular}

Source: compiled by the authors and from (') Behrouzi, Shaharoun, Ma'aram (2014).

In 47 balanced scorecard cases only 15 (32\%) had Kaplan \& Norton classic construction. Most of them were modified. This findings are similar to Wu\&Kuo (2012), Trotta et al. (2013), Behrouzi et al. (2014).

Just a few of research were followed by strategy map. Scopus database identified only 7 studies containing strategy map in hospital and all of them were linked to balanced scorecard.

In researches containing both: strategy map and balanced scorecard the perspectives are mostly similar to Kaplan \& Norton (Hong, 2008; Groene, 2009; Noh \& Seomun, 2015; Marquez et al., 2015). This result is surprising because in $68 \%$ of cases balanced scorecard were changed. Only in Verzola et al. (2009) publication strategic map perspectives were changed (Fig. 2) (Verzola et al., 2009).

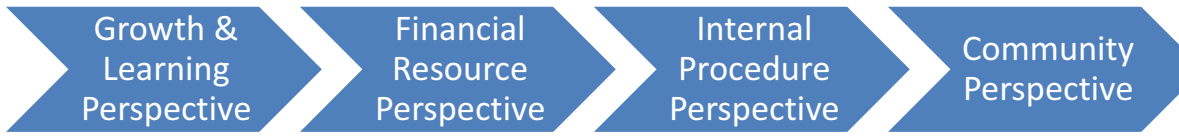

Figure 2. Strategy map perspectives of digestive endoscopy OU Ferrara University Hospital Source: Verzola et al., 2009.

What is important in this modification, that financials are the resource, not en effect (see Popesko et al., 2015).

The main hypothesis is that strategy map for hospital should differ from classic Kaplan \& Norton, because of different priorities, different fields of interests and hospital specificity. Strategy map can be also independent strategic management tool. 


\section{Methods}

The verification of hypothesis were made on "X" county hospital in Poland. Vision of hospital " $\mathrm{X}$ " is to provide medical care to the greatest number of people in need by providing high quality services, safety and build trust of the patient the whole society.

Mission concerns on values:

- good medicine doctor - highest quality of medical services,

- good employee - financial stability,

- good human - friendly to patients,

- good neighbour - active local community member.

For creation of strategy map goal intensity matrix (Białas, Czapiewski, 1995) were used. As a result of brainstorm session, management identified following critical objectives:

F1) Improve the profitability of health care services.

F2) Improve operational cash-flow.

F3) To fulfil contract with Third Party Payer (NFZ).

C1) Improvement of accessibility to medical services to residents of the municipalities.

C2) Improving the efficiency and professionalism in handling patients (patient satisfaction).

C3) Improving the quality of treatment (patient satisfaction).

I1) To maintain liquidity.

I2) Improve tangible fixed asset utilization.

I3) Improving the efficiency of use of Hospital inventory.

I4) Improve the performance of employees.

I5) Implementation of quality improvement programs.

L1) Continuous improvement of qualifications of staff.

L2) Keeping investments on housing base.

L3) The computerization of the hospital (implement electronic document flow).

L4) Introducing new methods of treatment and the purchase of modern equipment.

The next step was to build goal intensity matrix. Managers performed an examination of the interaction between all the objectives by using graphs.

\section{Results}

Tables was created with the objectives set during the study. Table of intensity was filled with values from 0 to 3 ( 0 interactions does not occur, 3 strong interactions) (Duraj, 2004). 


\begin{tabular}{|c|c|c|c|c|c|c|c|c|c|c|c|c|c|c|c|c|c|}
\hline & F1 & F2 & F3 & C1 & $\mathrm{C}^{2}$ & & C3 & I1 & 12 & 13 & 14 & 15 & L1 & L2 & L3 & L4 & $\begin{array}{l}\text { Acti } \\
\text { vity }\end{array}$ \\
\hline F1 & & 0 & 0 & 1 & 1 & 1 & 0 & 3 & 3 & 0 & 2 & 0 & 0 & 0 & 0 & 2 & 12 \\
\hline F2 & 1 & & 1 & & D & 0 & 1 & 3 & 1 & 1 & 2 & 1 & 2 & 3 & 3 & 3 & 22 \\
\hline F3 & 0 & 1 & & & D & 0 & 0 & 2 & 1 & 0 & 0 & 0 & 0 & 0 & 0 & 3 & 7 \\
\hline $\mathrm{C} 1$ & 0 & 1 & 2 & & & 2 & 3 & 0 & 0 & 0 & 1 & 0 & 0 & 0 & 0 & 0 & 9 \\
\hline $\mathrm{C} 2$ & 2 & 0 & 1 & & 3 & & 3 & 0 & 1 & 1 & 0 & 0 & 0 & 0 & 0 & 0 & 11 \\
\hline $\mathrm{C} 3$ & 0 & 0 & 1 & & 3 & 0 & & 0 & 0 & 0 & 0 & 1 & 0 & 0 & 0 & 0 & 5 \\
\hline I1 & 1 & 2 & 1 & & 1 & 0 & 0 & & 2 & 1 & 2 & 2 & 1 & 3 & 1 & 1 & 18 \\
\hline 12 & 3 & 3 & 0 & & 2 & 0 & 0 & 0 & & 2 & 2 & 0 & 0 & 0 & 0 & 1 & 13 \\
\hline 13 & 2 & 0 & 0 & & 2 & 1 & 0 & 0 & 0 & & 0 & 0 & 0 & 0 & 0 & 2 & 7 \\
\hline 14 & 2 & 2 & 0 & & ) & 0 & 2 & 2 & 2 & 0 & & 0 & 0 & 0 & 0 & 0 & 10 \\
\hline 15 & 0 & 0 & 0 & & D & 2 & 0 & 0 & 2 & 2 & 3 & & 0 & 0 & 2 & 2 & 13 \\
\hline L1 & 0 & 0 & 0 & ) & 3 & 3 & 3 & 0 & 0 & 0 & 0 & 0 & & 0 & 0 & 2 & 11 \\
\hline L2 & 3 & 0 & 0 & & 2 & 1 & 3 & 0 & 2 & 3 & 0 & 0 & 0 & & 0 & 3 & 17 \\
\hline L3 & 0 & 0 & 0 & & D & 0 & 0 & 0 & 0 & 0 & 3 & 3 & 0 & 0 & & 1 & 7 \\
\hline L4 & 2 & 0 & 3 & 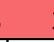 & 3 & 0 & 0 & 0 & 1 & 0 & 1 & 0 & 0 & 0 & 0 & & 10 \\
\hline $\begin{array}{l}\text { Rea } \\
\text { ctivi } \\
\text { ty }\end{array}$ & 16 & 9 & 9 & 2 & & 10 & 15 & 10 & 15 & 10 & 16 & 7 & 3 & 6 & 6 & 20 & \\
\hline
\end{tabular}

Figure 3. Goal intensity matrix Source: own.

Connecting points of with the strongest interactions (3) - strategy map has been created (Fig. 4).

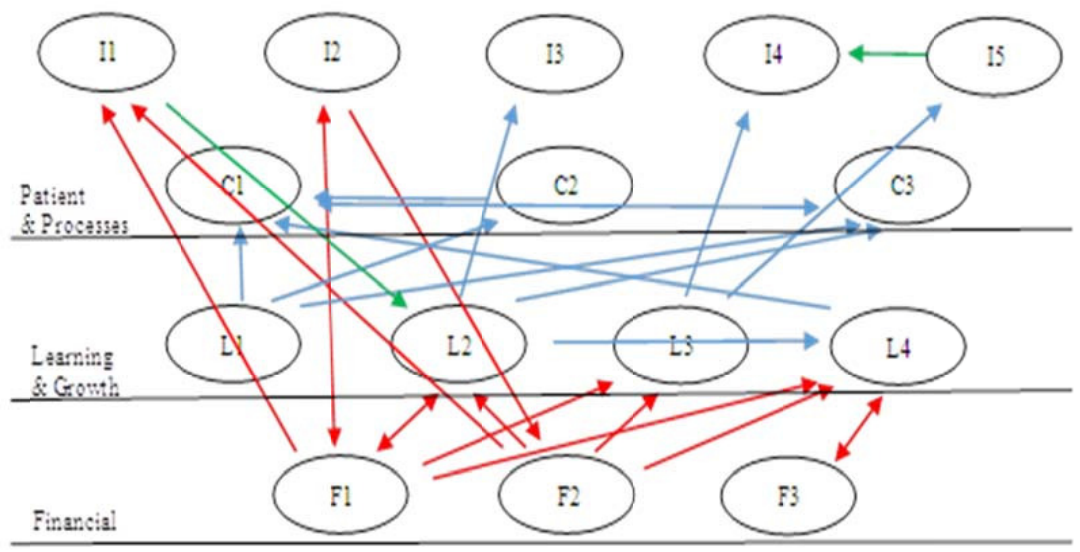

Figure 4. Strategy map for "X" County Hospital in Poland Source: own.

The financial perspective is fundamental, because it implicates many other objectives, and is implicated by just a few of them (red arrow direction). Improve the profitability of health care services, improve operational cash-flow, and to fulfil contract with Third Party 
Payer (NFZ) have great impact of economic situation of the hospital. Economic situation allows to realize objectives in other perspectives.

Learning \& growth perspective have objectives connected with building technical, organization and human potential of hospital. This objectives are: continuous improvement of qualifications of staff, keeping investments on housing base, computerization of the hospital (implement electronic document flow), introducing new methods of treatment and the purchase of modern equipment. Growth of hospital potential depends of financial situation and implicates quality of processes and patient satisfaction.

Patient \& processes perspective is connected with medical treatment process. This is the result of hospital activity. The good results of hospital activity should be visible i.e. in improvement of accessibility to medical services to residents of the municipalities, improving the efficiency and professionalism in handling patients (patient satisfaction), improving the quality of treatment (patient satisfaction), improving the efficiency of use of Hospital inventory, improve the performance of employees, implementation of quality improvement programs.

\section{Conclusion}

How strategy map changes hospital management? First of all, strategy map is useful tool to visualize objectives. This visualization reflects priorities and express connection between objectives. What is critical, that strategy map is different in hospitals then in other organizations. This is mainly because hospital is an organization realizing not only economic aims, but also this connected with sustainable development. Various range of objectives needs a specific strategic management tool.

Strategic map of "X" County Hospital in Poland have following structure (Fig. 5).

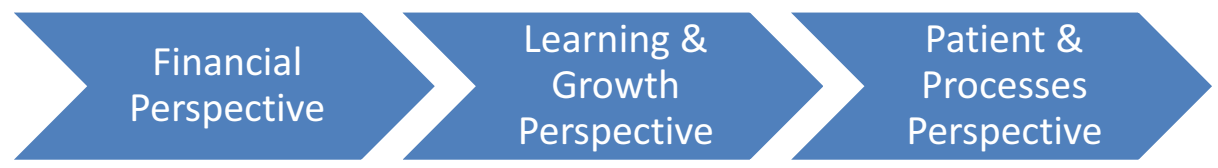

Figure 5. Strategy map perspectives of " $\mathrm{X}$ " county hospital in Poland Source: own.

The financial perspective is fundamental, because it implicates many other objectives (see Korpysa, 2013). Good financial condition allow to invest in inventory, human resources and new medical procedures, but this have sense, when reflects results in patient \& processes perspective (see Heurich \& Vignali, 2015), i.e. will increase accessibility to medical services, improve quality of treatment, efficiency of employees.

Strategy map in this form can enable collaboration between the various organizational figures, to realize business strategy which was accessible to the health workers involved. It brings the business strategy to the objectives attributed to each operative unit. Nevertheless, strategy map is the tool orientated to guide managerial action. Strategy may be communicated to all levels of the organization, and allocation of resources can reflect the strategic aims.

Farther research will concentrate on integration strategy map to other strategic management tools. 


\section{References}

Abo-Hamad, W., Arisha, A. (2013), Simulation-based framework to improve patient experience in an emergency department, European Journal of Operational Research, 224 (1), pp. 154-166.

Baraldi, S., Cifalinò, A. (2015), Delivering training strategies: the balanced scorecard at work, International Journal of Training and Development, Vol. 19, Issue 3, pp. 179-198, September.

Behrouzi, F., Shaharoun A.M., Ma'aram A. (2014), Applications of the balanced scorecard for strategic management and performance measurement in the health sector, Australian Health Review, 38, pp. 208-217.

Bernstein, A. B., Hing, E., Moss, A. J., et al. (2003), Health care in America: Trends in utilization, Hyattsville, Maryland, National Center for Health Statistics.

Białas, T., Czapiewski, M. (1995), Ustalanie zależności celów w procesie budowania strategii przedsiębiorstwa, Przeglad Organizacji, No. 10, pp. 16-18.

Biazzo, S., Garengo, P. (2012), Performance Measurement with the Balanced Scorecard. A Practical Approach to Implementation within SMEs, Springer, Heidelberg.

Bouland, D. L., Fink, E., Fontanesi, J. (2011), Introduction of the balanced scorecard into an academic department of medicine: Creating a road map to success, Journal of Medical Practice Management, 26 (6), pp. 331-335.

Cattinelli, I., Bolzoni, E., Barbieri, C. et al. (2012), Use of Self-Organizing Maps for Balanced Scorecard analysis to monitor the performance of dialysis clinic chains, Health Care Management Science, 15 (1), pp. 79-90.

Duraj, J. (2004), Podstawy ekonomiki przedsiębiorstw, PWE, Warszawa, pp. 51-58.

Ermakova, S. E., Izmalkov, N. S., Evdokimova, E. G. (2014), Evaluation of the economic efficiency of process-oriented management system at healthcare organizations, European Journal of Physical and Health Education, 6, BM-012-14.

Glouberman, S., Mintzberg, H. (2001), Managing the care of health and the cure of diseasePart I: Differentiation, Health Care Manage Rev., Winter 26(1), pp. 56-69.

Grigoroudis, E., Orfanoudaki, E., Zopounidis, C. (2012), Strategic performance measurement in a healthcare organisation: A multiple criteria approach based on balanced scorecard, Omega, 40 (1), pp. 104-119.

Groene, O., Brandt, E., Schmidt, W., Moeller J. (2009), The Balanced Scorecard of acute settings: development process, definition of 20 strategic objectives and implementation, International Journal for Quality in Health Care, Vol. 21, No. 4, pp. 259-271.

Gutiérrez López, C., Culebras, J. L. M. J. (2015), El cuadro de mando integral como instrumento de gestión en la nutrición clínica, Nutr Hosp., 32(1), pp.403-410.

Heurich, M., Vignali, C. (2015), Innovations and its Impact on the Performance of Acute Care Hospitals in Germany - An Investigation Containing Empirical Research and Software Development, Economics and Sociology, Vol. 8, No 4.

Hong, Y., Hwang, K. J., Kim, M. J., Park, C. G. (2008), Balanced Scorecard for Performance Measurement of a Nursing Organization in a Korean Hospital, J Korean Acad Nurs, Vol. 38, No. 1, pp. 45-54.

Hopwood, B., Mellor, M., O’Brien, G. (2005), Sustainable Development: Mapping Different Approaches, Sustainable Development, 13, pp. 38-52.

Kaplan, R. S., Norton D. P. (2000), Having Trouble with Your Strategy? Then Map it, Harvard Business Review, September-October, p. 176.

Kaplan, R. S., Norton D. P. (2004), Strategy Maps. Converting Intangible Assets Into Tangible Outcomes, Harvard Business School Press, Boston. 
Khan, M. M. R., Hotchkiss, D., Dmytraczenko T., Zunaid A. K. (2013), Use of a Balanced Scorecard in strengthening health systems in developing countries: An analysis based on nationally representative Bangladesh Health Facility Survey, International Journal of Health Planning and Management, 28 (2), pp. 202-215.

Korpysa, J. (2013), Buyer behaviour in the context of sustainable consumption policy pursued in Poland, Amfiteatru Economic, Vol. XV, Special No. 7, pp. 702-713.

Lin, Q. L., Liu, L., Liu, H. C., Wang, D. J. (2013), Integrating hierarchical balanced scorecard with fuzzy linguistic for evaluating operating room performance in hospitals, Expert Systems with Applications, 40 (6), pp. 1917-1924.

Lovaglio, P. G. (2010), Model building and estimation strategies for implementing the Balanced Scorecard in Health sector, Quality and Quantity, 45 (1), pp. 199-212.

Luigi, D., Iuliana, C., Alma, P., Bilan, Y. (2014), Directly estimating the private healthcare services demand in Romania, Journal of International Studies, Vol. 7, No 3, pp. 55-69.

Marquez, P. B., Barroso, C. C. A., Silva A. M. et al. (2015), Proposta de medidas de avaliação de desempenho para o Centro de Engenharia Clínica e Bioequipamentos do HCFMRPUSP, Medicina (Ribeirão Preto), 48(1), pp. 65-76.

Noh, W., Seomun, G. A. (2015), Development and Application of a Performance Prediction Model for Home Care Nursing Based on a Balanced Scorecard using the Bayesian Belief Network, J Korean Acad Nurs, Vol. 45, No. 3, pp. 429-438.

Popesko, B., Novák, P., Papadaki, S. (2015), Measuring diagnosis and patient profitability in healthcare: economics vs ethics, Economics and Sociology, Vol. 8, No. 1, pp. 234-245.

Rabbani, F., Lalji, S. N. H., Abbas, F. et al. (2011), Understanding the context of balanced scorecard implementation: A hospital-based case study in Pakistan, Implementation Science, 6 (1), 31, pp. 1-14.

Rabbani, F., Pradhan, N. A., Zaidi, S., Azam, S. I., Yousuf, F. (2015), Service quality in contracted facilities, International Journal of Health Care Quality Assurance, pp. 520531.

Rudawska, I. (2013), Sieć jako pośrednia forma koordynacji gospodarczej na przykładzie zintegowanej opieki zdrowotnej, Prace Naukowe Uniwersytetu Ekonomicznego We Wrocławiu, 307, pp. 513-521.

Rudawska, I., Kaufmann, H. R. (2015), A quality management model for integrated healthcare in Poland, World Review of Entrepreneurship, Management and Sustainable, Vol. 11, Issue 2/3, pp. 247-266.

Rudawska, I., Pętlak, M. (2013), Improving the efficiency of health care in Poland conceptual information flow framework, Business Management Dynamics, Vol. 2, No. 8, Feb., pp. 31-38.

Rudawska, I., Urbańczyk, E. (2014), Opieka zdrowotna. Zagadnienia ekonomiczne, Difin, Warszawa.

Smith, C., Christiansen T., Dick D. et al. (2014), Performance management tools motivate change at the frontlines, Healthcare Management Forum, 27 (1), pp. 15-19.

Steinke, C., Webster, L., Fontaine, M. (2010), Evaluating building performance in healthcare facilities: An organizational perspective, Health Environments Research and Design Journal, 3 (2), pp. 63-83.

Sustainable Development and Corporate Social Responsibility, http://work.chron.com (referred on 30.12.2015).

Szabo, S., Sidor, J. (2014), The performance measurement system-potentials and barriers for its implementation in healthcare facilities, Journal of Applied Economic Sciences, 9 (4), pp. 728-735.

Świerk, J. (2009), Mapa strategii i strategiczna karta wyników w planowaniu działań przedsiębiorstwa, UMCS, Lublin, pp. 94-107. 
Top health industry issues of 2015. Outlines of a market emerge (2014), Health Research Institute, PWC, Dec.

Top health industry issues of 2016, Health Research Institute (2015), PWC, Dec.

Transforming our world: the 2030 Agenda for Sustainable Development, United Nation, 25 September 2015, A/70/L.1

Trotta, A., Cardamone, E., Cavallaro, G., Mauro, M. (2013), Applying the Balanced Scorecard approach in teaching hospitals: a literature review and conceptual framework, Int J Health Plann Mgmt, 28, pp. 181-201.

Urbaniec, M. (2015), Towards Sustainable Development through Ecoinnovations: Drivers and Barriers in Poland, Economics and Sociology, Vol. 8, No 4, pp. 179-190.

Verzola, A., Bentivegna, R., Carandina, G. (2009), Multidimensional evaluation of performance: experimental application of the balanced scorecard in Ferrara university hospital, Cost Effectiveness and Resource Allocation, 7 (15), pp. 1-5.

Wu, I.-L., Kuo Y.-Z. (2012), A Balanced Scorecard Approach in Assessing IT Value in Healthcare Sector: An Empirical Examination, J Med Syst, 36, pp. 3583-3596. 